\title{
Review
}

\section{Reading between the genetic lines: How epigenetics is unlocking novel therapies for type 1 diabetes}

\author{
Ammira-Sarah Akil *, Laila F. Jerman, Esraa Yassin, Sujitha Subash Padma Jeya, Alya Al Kurbi, \\ and Khalid Fakhro \\ Precision Medicine Program, Research Department, Sidra Medicine, Qatar; aakil@sidra.org, Lali@sidra.org, \\ esraayassin@gmail.com, ssubashpad@sidra.org, aalkurbi2@sidra.org, kfakhro@sidra.org \\ * Correspondence: Ammira-Sarah Akil; aakil@sidra.org; Tel.: (+97450597431)
}

\begin{abstract}
Type 1 diabetes (T1D) is an autoimmune condition where the body's immune cells destroy their insulin-producing pancreatic beta-cells leading to dysregulated glycaemia. Individuals with T1D control their blood glucose through exogenous insulin replacement therapy, often using multiple daily injections or pumps. However, failure to accurately mimic intrinsic glucose regulation results in glucose fluctuations and long-term complications impacting key organs such as the heart, kidneys, and/or the eyes. It is well-established that genetic and environmental factors contribute to the initiation and progression of type 1 diabetes, but recent studies show that epigenetic modifications are also important. Here, we discuss key epigenetic modifications associated with type 1 diabetes pathogenesis and discuss how recent research is finding ways to harness epigenetic mechanisms to prevent, reverse, or manage type 1 diabetes.
\end{abstract}

Keywords: Chromatin; DNA methylation; Epigenetics; Histone modifications; Metaboloepigenetics; miRNA; Therapy; Type 1 diabetes

\section{Abbreviations:}

CTLA4: Cytotoxic T-lymphocyte associated protein 4

DNMT: DNA methyltransferase

HDAC: Histone deacetylases

HLA-DRB1: Major histocompatibility complex, class II, DR Beta 1

HLA-DQB1: Major histocompatibility complex, class II, DQ Beta 1

MZ: $\quad$ Monozygotic

\section{Introduction}

The hallmark of Type 1 diabetes (T1D) is T-cell mediated autoimmune destruction of pancreatic beta-cells, leading to insulin deficiency, elevated blood glucose concentrations, and life-long need for exogenous insulin therapy. Globally, the annual number of new cases of type 1 diabetes is rising, and in the under-twenties age group alone, numbers are fast approaching 100,000 (www.diabetesatlas.org). The incidence rate varies between countries and ranges from $6 \%$ in subSaharan Africa to $77 \%$ in some parts of Scandinavia [1]; altogether another five million people are expected to be diagnosed with type 1 diabetes by 2050 [2], with profound implications for healthcare systems globally and the potential for staggering socio-economic impact. Staying well with type 1 diabetes requires normoglycaemia to be achieved and maintained, but this goal is not currently achievable for many patients using existing treatment strategies [3]. Understanding the intrinsic and extrinsic factors underlying the development and progression of type 1 diabetes is necessary for the development of novel intervention therapies that could delay or even prevent the clinical progression 
in individuals susceptible to T1D or be used to treat/manage glycemic excursions in those worst affected.

Despite ongoing research, dissecting the etiology of type 1 diabetes has proven a herculean task. Genetic studies have recognized more than 60 regions (loci) associated with or predisposing for type 1 diabetes; the first and still strongest reported genetic association is with the human leukocyte antigen HLA region [4-6]; while the second most significant type 1 diabetes genetic association is with insulin gene promotor polymorphism [7]. Even so, only $5 \%$ or fewer of children bearing the high-risk HLA haplotypes and about $10 \%$ with insulin gene polymorphism go on to clinically develop type 1 diabetes during the first 20 years of their lifetime [8,9]. So what are the important factors determining whether a genetically-susceptible individual develops type 1 diabetes, and how could these factors be used to help in the fight against this disease?

Epigenetic modification is a key mechanism affecting gene expression - effectively determining the extent and timing of expression of a given gene in response to internal and external stimuli. Multiple lines of evidence now implicate that epigenetic factors in type 1 diabetes' incidence: firstly, although monozygotic (MZ) twins share 100\% of their DNA they exhibit a high discordance rate in the development of type 1 diabetes, especially when onset occurs over the age of 15 [10]; furthermore, the rapid increase in type 1 diabetes seen in recent years is unlikely to be a result of genetic changes across the population alone due to the short timescale [11, 12]. Alongside, other studies showed that in siblings sharing an HLA haplotype it is rather the age at diabetes onset in their sibling that determines their risk of developing the condition, and not the shared HLA type itself [13]. Thus it is not only the possession of high-risk genes that decide whether or not an individual progresses to the clinical presentation of type 1 diabetes but rather whether, when, and to what extent those genes are expressed.

The growing interest in epigenetic changes in type 1 diabetes and factors driving them has led to investigations into epigenetic therapies to treat the condition. In this review, we discuss recent initiatives targeting epigenetic mechanisms involved in type 1 diabetes, namely DNA methylation, histone (post-translational) modifications, and non-coding RNA-mediated gene silencing (for a comprehensive discussion of these mechanisms see [14, 15]), as well as highlighting future avenues for productive therapeutic research.

\section{Therapies targeting DNA methylation in type 1 diabetes}

DNA methylation involves the addition of a methyl group to the fifth carbon of CpG dinucleotides (cytosine residues linked by a phosphate to a guanine nucleotide) by DNA methyltransferases, thereby blocking gene transcription [16] (Figure 1). In type 1 diabetes there is evidence that DNA methylation in both immune cells and pancreatic islet cells is important, and may even substantially pre-date diagnosis of the disease (reviewed in [17]). Studies of immune cells from the blood of adult MZ twins discordant for type 1 diabetes have variably identified specific positions with distinct methylation patterns: one study on monocytes found that patterns of methylation at these sites were detectable many years before T1D diagnosis and were correlated with the presence of autoantibodies [18]; while a genome-wide DNA methylation analysis identified $88 \mathrm{CpG}$ methylation sites in B-lymphocytes with significant modification, including some affecting genes associated with type 1 diabetes pathogenesis including HLA, INS, IL-2RB and CD226 [19]. A more recent MZ twin study did not identify any differences in DNA methylation in monocytes or B-cells but found a single differentially-methylated $\mathrm{CpG}$ position in T-cells with genome-wide significance [20].

Genome-wide DNA methylation quantitative trait locus (mQTL) analysis of human pancreatic islets has revealed 383 significant $\mathrm{CPG}$ sites in known diabetes loci as potential methylation targets; importantly, some of the identified candidate genes (GPX7, GSTT1, and SNX19) directly affect key biological processes such as proliferation and apoptosis of pancreatic islet beta-cells [21]. More recently, Ye et al. identified widespread genetic and epigenetic interactions at known type 1 diabetes susceptibility loci; they showed (in their preprint) that DNA methylation at five loci; ITGB3BP, AFF3, PTPN2, CTSH, and CTLA4, is potentially causal to type 1 diabetes [22]. Interestingly, it seems that 
some epigenetic changes may even be present years before diagnosis: Johnson et al. identified 10 regions of the genome in which methylation differs between type 1 diabetes cases and controls from as young as birth, in some cases multiple years before T1D diagnosis [23]. These findings raise the possibility of screening those with high-risk genetic phenotypes for concurrent high-risk epigenetic marks to identify those at greatest need of intensive monitoring and/or preventative intervention.

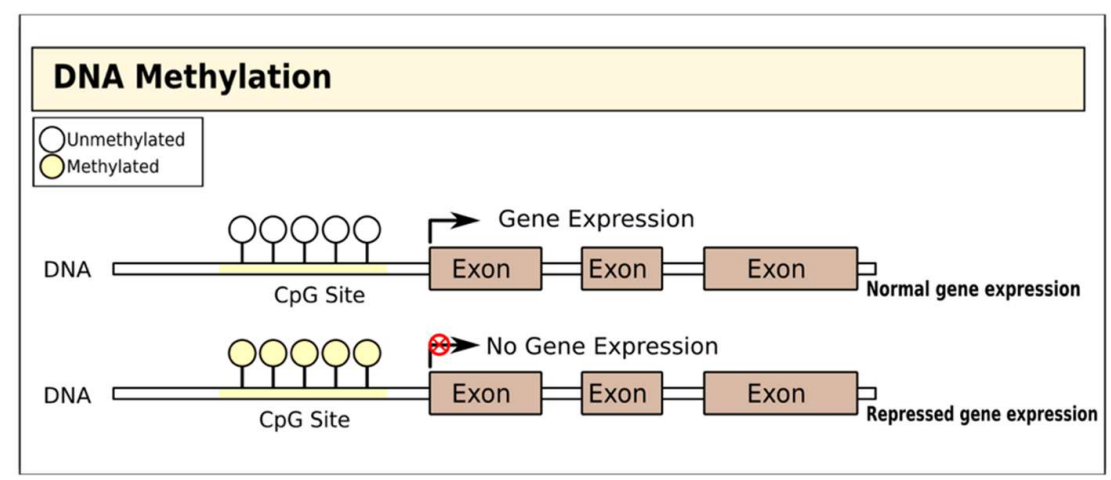

Figure 1 Gene regulation by DNA methylation. DNA is methylated by the covalent addition of a methyl group to $\mathrm{CpG}$ dinucleotides by DNA methyltransferases. This process is generally associated with gene silencing.

Although the significance of altered DNA methylation in type 1 diabetes onset and pathogenesis is clear, there have yet to be large-scale trials of the use of DNA methyltransferase inhibitors in treating/preventing the condition. However, inhibitors of DNA methylation have been studied in vitro for their potential use in pancreatic cell reprogramming with the future aim of regenerating patients' beta-cells as a novel type 1 diabetes therapy. Lefebvre et al. found that treating pancreatic ductal cells with the DNA methylation inhibitor 5-azacytidine successfully promoted their conversion into pancreatic endocrine cells [24]. Similarly, in mice lacking DNA methyltransferase 1 and the alpha-cell maintaining factor Arx specifically in pancreatic alpha-cells, Chakravarthy et al. observed reprogramming of around half of alpha-cells into biologically active beta-cells in vivo [25]. Interestingly, these authors also found that a subset of type 1 diabetes patients possess a small number of glucagon-expressing cells lacking both DNA methyltransferase 1 and Arx expression, and instead ectopically express beta-cell markers, including insulin [25]. While these results suggest that targeted DNA methyltransferase inhibition might support the possibility of regeneration of lost betacells in individuals with type 1 diabetes, further studies addressing the longevity and functionality of these converted cells are warranted.

While not specifically assessing epigenetic modification, there are early indications that drugs as well as diets modulating DNA methylation could have some positive effect on delaying the progression to type 1 diabetes. Long used in diabetes care, metformin has more recently been revealed to have multiple epigenetic modifying actions, including modulating the activity of DNA methyltransferases [26]. The REducing with MetfOrmin Vascular Adverse Lesions (REMOVAL trial) aimed to ameliorate long-term cardiovascular complications in diabetes patients using metformin [27]. In the REMOVAL trial, metformin treatment reduced the progression of atherosclerosis, weight gain, and LDL-cholesterol levels [28, 29], though the epigenetic effects of treatment were not specifically measured. In type 2 diabetes metformin treatment significantly decreased DNA methylation of metformin transporter genes (SLC22A1, SLC22A3, and MATE1) in the livers of patients; in the case of SLC22A1 and SLC22A3, reduced methylation was associated with less hyperglycemia and obesity [30]. Similarly, promising results were also seen in pre-clinical trials in adult rats with streptozotocin-induced diabetes treated with the DNMT inhibitor procainamide, which significantly reduced the activity of DNMT in the pancreas and significantly increased fasting insulin levels [31]. Thus, initial studies in both type 1 and 2 diabetes patients and models seem to 
indicate that DNMT inhibitors might be a promising therapeutic strategy. Several DNMT inhibitors are licensed for use in treating certain types of cancer [32] and could be worth exploring in diabetes.

\section{Therapies targeting histone modifications in type 1 diabetes}

Alongside direct epigenetic modification of DNA, histones undergo post-transcriptional modifications to restructure chromatin in different ways [33-37]. Genomic DNA is bundled into chromatin, creating nucleosomes; the histone tails project from the nucleosome and are exposed to a wide range of covalent modifications including methylation, acetylation, ubiquitination, phosphorylation, sumoylation, and ADP ribosylation [38, 39]. Collectively, these post-transcriptional modifications act to regulate chromatin structure, which has profound effects on several biological activities including gene transcription, DNA repair, and chromosome condensation [33]. (Figure 2).

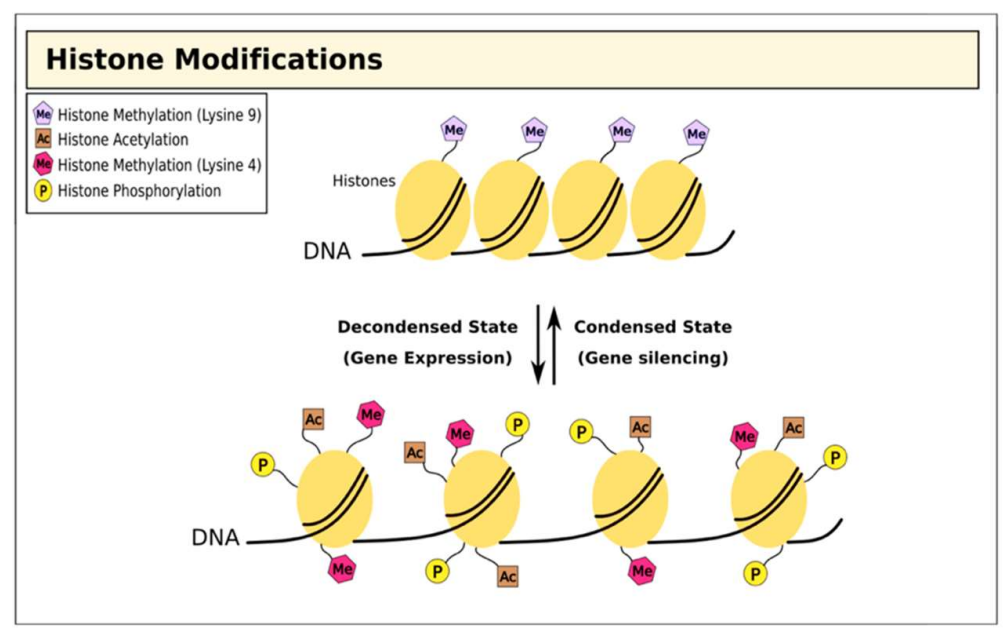

Figure 2. Histone modifications and chromatin structure. Histone methylation at lysine residues is associated with both gene expression and silencing, while acetylation is associated with repression.

Histone modification is a significant feature of type 1 diabetes (reviewed in [40-42]), with studies showing aberrant histone modification as well as differential expression of the histone-modifying enzymes histone methyltransferase and histone deacetylase. A pivotal study by Miao et al. revealed increased methylation of lysine 9 in the $\mathrm{H} 3$ histone protein (H3K9me2) in lymphocytes from type 1 diabetes patients: importantly, several of the genes affected had been linked with type 1 diabetes pathology, such as CLTA4, a known type 1 diabetes susceptibility gene, as well as genes affecting the signaling pathways of the immune mediators transforming growth factor- $\beta$, nuclear factor-kB, p38 mitogen-activated protein kinase, toll-like receptors, and interleukin-6 [43]. The same group went on to show that acetylation of lysine 9 in the $\mathrm{H} 3$ histone protein (H3K9Ac) upstream of major type 1 diabetes susceptibility genes $H L A-D R B 1$ and $H L A-D Q B 1$ was also significantly higher in monocytes from patients than from controls [44]. Furthermore, differential histone methylation and acetylation might be one mechanism explaining the type 1 diabetes discordance seen in $\mathrm{MZ}$ twin studies. Following exposure to high glucose levels in vitro skin fibroblasts showed significantly different expression of genes regulating epigenetic processes depending on whether they came from the affected or unaffected twin; while expression of histone lysine methyltransferase (SET 7), H3K4 methyltransferase, and histone deacetylase (HDAC) 8 was lower in the cells from the type 1 diabetic twin, HDAC 4 was expressed at a higher level [45].

Histone modification inhibitors are a well-characterized class of epi-drugs with clear potential to be used therapeutically in type 1 diabetes, either alone or in combination with DNA methyltransferase inhibitors. HDAC inhibitors (HDACi) were investigated by Lundh et al. after finding that pancreatic beta-cells from children with newly-diagnosed type 1 diabetes exhibited 
relatively higher levels of HDAC1 and lower levels of HDACs -2 and -3 compared to controls; investigating this further in vitro the authors showed that inhibition of HDAC1 and -3, but not HDAC2, protected primary and immortalized rat beta-cells from apoptosis due to inflammatory cytokine exposure [46]. Building on this work, treating non-obese diabetic mice with the licensed lysine deacetylase inhibitors vorinostat and givinostat reversed diabetes [47], calling for future trials in human patients. Similarly encouraging data has come from trials of valproic acid, an HDAC class I and II inhibitor: in juvenile rats, valproic acid treatment positively influenced beta-cell proliferation and functionality and increased insulin production, while simultaneously decreasing beta-cell apoptosis, HBA1c and plasma glucose levels [48, 49]. Studies in murine models also showed that the small molecule HDAC inhibitor trichostatin A [50] prevented type 1 diabetes symptoms through increased acetylation of histone $\mathrm{H} 3$ which was associated with increased expression of the $\mathrm{CD} 4{ }^{+} \mathrm{T}$ cell-derived lymphokine (Ifng) and its transcription factor Tbet/Tbx21 [51], highlighting the potential role of chromatin remodeling agents in protection against the development of type 1 diabetes.

HDACi have also been used in studies of therapeutic cell reprogramming in the hope that this might be translated into a clinical application. Similarly to DNA methylation inhibitors, in vitro inhibition of the H3K27me3 methyltransferase Ezh2 enhanced Neurogenin3-mediated production of beta-like cells from human embryonic stem cells [52], as well as the formation of insulin-producing cells from human induced pluripotent cells [53]. Combining the HDACi romidepsin with the DNA methyltransferase inhibitor 5-azacytidine also led to improved differentiation of adult human dermal fibroblasts into insulin- and glucagon-producing cells [54], but again the therapeutic translation of these findings is so far lacking. Recent studies from Marino E, and colleagues [55] demonstrate that feeding NOD mice a combination of acetate- and butyrate-rich diets offered protection against T1D progression. These studies indicate that acetate and butyrate might be working through distinct mechanisms and that a beneficial effect may be obtained through a combination of diets rich in these short-chain fatty acids. Acetate decreased the frequency of autoreactive T-cells, whilst butyrate increased the number and function of regulatory T-cells. Acetate- and butyrate-yielding diets enhanced gut integrity, reduced circulating concentration of diabetogenic cytokines (such as IL-21) and demonstrated prevention in T1D onset. Clinical trials using such diets will provide intriguing results in understanding the timing and potential of such diets in individuals at high risk of T1D.

\section{Therapies targeting microRNAs in type 1 diabetes}

MicroRNAs (miRNAs) are short (around 22 nucleotides long) non-coding single-stranded RNA molecules [56] that modulate post-transcriptional gene expression, leading to target mRNA silencing (Figure 3). Multiple studies have demonstrated important associations between specific miRNAs and type 1 diabetes (reviewed in [57]). One study on children with recent-onset type 1 diabetes identified six miRNAs (miR-454-3p, miR-222-3p, miR-144-5p, miR-345-5p, miR-24-3p, and miR-140-5p) with high expression only in the early stages of diabetes onset; moreover, pathway analysis associated this pattern of differential miRNA expression with glycosaminoglycan biosynthesis as well as PI3K/Akt, MAPK, and Wnt signaling pathways in early type 1 diabetes [58]. Similarly, we also demonstrated that enteroviral Infections disrupt the miRNA-directed suppression of proinflammatory factors within human islet beta-cells, thereby resulting in an exacerbated antiviral immune response that promotes beta-cell destruction and eventual type 1 diabetes [59]. Further research on pancreatic cells from type 1 diabetes patients linked expression of miR-23a-3p, miR-23b-3p, and miR-149-5p with that of proapoptotic Bcl-2 proteins and consequent human $\beta$-cell apoptosis [60]; while autoreactive CD8 ${ }^{+}$ T-cells from patients with type 1 diabetes showed evidence of repression of pro-apoptotic pathways via increased expression of miR-23b, miR-98 and miR-590-5p [61]. Thus miRNAs are implicated at every stage of type 1 diabetes, leading to intense research interest in targeting these molecules therapeutically. 


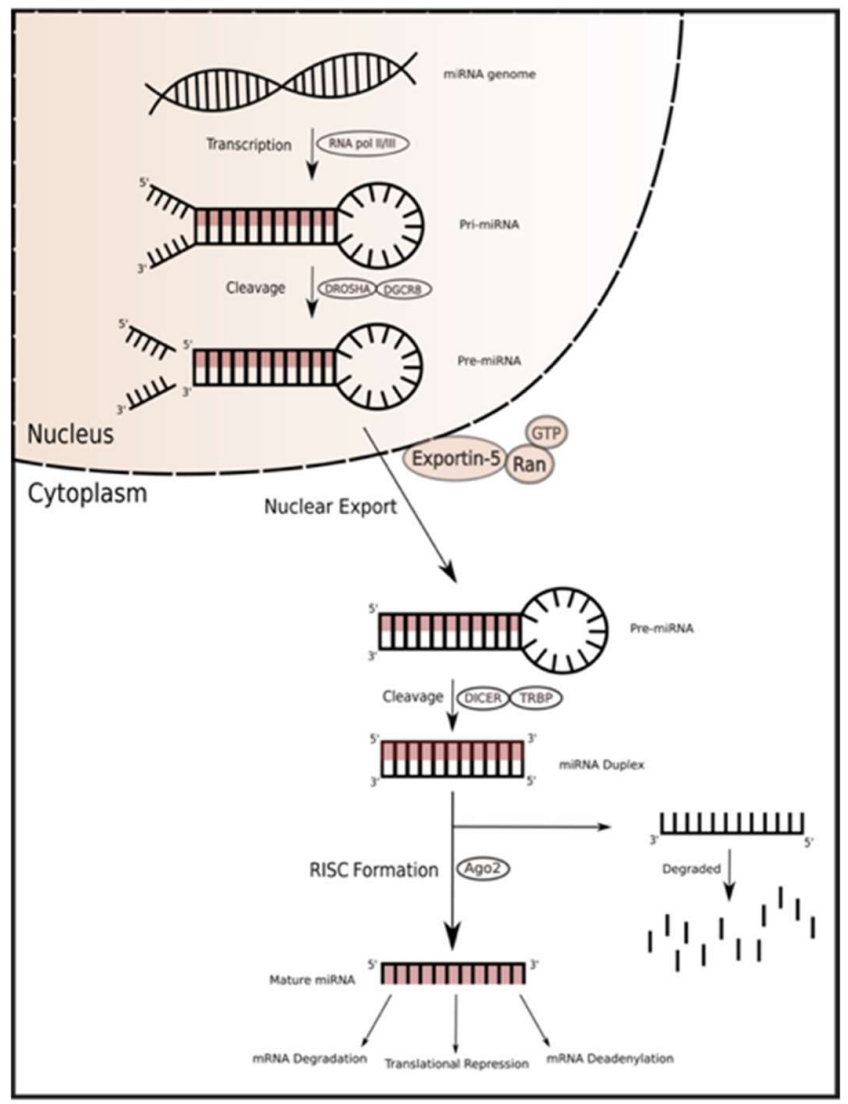

Figure 3 MiRNA biogenesis and mechanism of action. MiRNAs are transcribed in the nucleus as primary miRNA (pri-miRNA) then cleaved into precursor miRNA (pre-miRNA) by the DORSHADGCR8 microprocessor complex. Pre-miRNA is then processed in the cytoplasm by the DICER enzyme forming the bioactive miRNA duplex which can be bound by the RNA-induced silencing complex [62] ready to target the complementary mRNA for silencing.

To date, the majority of studies exploring the therapeutic potential of miRNA-targeted treatments for type 1 diabetes have been carried out in vitro; still, some have yielded promising insights. El Ouaamari et al. showed that the application of 20 -O-methyl-miR-375 antisense oligonucleotides increased expression of the target gene PDK1 and reverted insulin release to normal in the rat beta-cell line INS-1E [63]. Another study found that blocking miR-21, miR-34a or miR-146a functions with antisense molecules preserved glucose responsiveness in the murine pancreatic betacell line MIN6 under treatment with the inflammatory cytokine IL1 $\beta$ treatment, and also protected these cells from cytokine-induced apoptosis [64]; while Lovis et al. showed that blocking miR-34a or miR-146 activity partially protected palmitate-treated MIN6 cells from apoptosis but was insufficient to restore normal insulin secretion [65]. A major challenge of the clinical application of miRNA targeting is delivering the therapeutic agent to the target cells at an effective concentration. Pileggi et al. made important progress in this regard by establishing an islet transplantation model in rats where fluorescently-labeled miRNA inhibitors were directly infused via an implanted catheter, and effectively localized within target cells [66]. This study paves the way for in vivo trials of miRNA inhibitors aiming to preserve beta-cell function or to increase the efficacy of transplantation of autologous islets or those generated from induced pluripotent stem cells. Building on such work, a recent study conducted by Wang et al demonstrated the potential of miR-216a to act as a therapeutic target in type 1 diabetes through its negative regulation of phosphatase and tensin homolog (PTEN) 
expression levels; administration of a miR-216a mimic in a murine model of type 1 diabetes increased beta-cell proliferation, decreased PTEN expression, and improved insulin secretion in vivo [67].

Despite these promising results and encouraging progress, many obstacles remain on the path to realizing the potential of miRNA-targeted therapies in type 1 diabetes. For example, some miRNAs have multiple target genes, and occasionally, multiple miRNAs can regulate the same gene: this complex regulatory network can control several genes via one or a combination of miRNAs, further complicated by interactions with other epigenetic factors and linkers, such as DNA methylation and the histone code $[68,69]$. Future research will require the current studies on miRNA at the cellular level to be extended into well-characterized type 1 diabetes in vivo models to understand the direct role of miRNA-targeted genes and therapies in diabetes treatment.

Table 1. miRNAs that are differentially expressed between type 1 diabetes patients and unaffected controls.

\begin{tabular}{|c|c|c|c|c|}
\hline miRNA & $\begin{array}{l}\text { Target } \\
\text { gene/pathway } \\
\text { related to T1D }\end{array}$ & $\begin{array}{l}\text { Regulation T1D } \\
\text { patients/controls }\end{array}$ & Tissue/cell & Reference \\
\hline miR-101-3p & $\begin{array}{l}\text { c-Met-HGF, ephrin } \\
\text { receptor, and STAT3 } \\
\text { pathways linked to } \\
\text { insulin secretion } \\
\text { and } \beta \text { cells survival }\end{array}$ & Upregulated & Serum & [70] \\
\hline $\begin{array}{l}\text { miR-125b- } \\
5 p \\
\text { and miR- } \\
365 a-3 p\end{array}$ & $\begin{array}{l}\text { variation of } \\
\text { hyperglycemia, } \\
\text { measured (HbA1c) }\end{array}$ & Upregulated & Plasma & [71] \\
\hline $\begin{array}{l}\text { miR-5190 } \\
\text { and } \\
\text { miR- } 770- \\
5 p\end{array}$ & $\begin{array}{l}\text { variation of } \\
\text { hyperglycemia, } \\
\text { measured (HbA1c) }\end{array}$ & Downregulated & Plasma & [71] \\
\hline miR-100-5p & $\begin{array}{l}\text { Wnt signaling } \\
\text { pathway }\end{array}$ & Downregulated & Serum & [58] \\
\hline miR-150-5p & $\begin{array}{l}\text { transcription factor } \\
\text { c-Myb and IFN-r }\end{array}$ & Downregulated & PBMCs & [72] \\
\hline
\end{tabular}




\begin{tabular}{|c|c|c|c|c|}
\hline miR-154-3p & $\begin{array}{l}\text { glycosaminoglycan } \\
\text { biosynthesis }\end{array}$ & Downregulated & Serum & [58] \\
\hline miR-24-3p & $\begin{array}{l}\text { MAPK and Wnt } \\
\text { signaling pathways }\end{array}$ & $\mathrm{Up}$ & Serum & [58] \\
\hline miR-25-3p & $\begin{array}{ll}\text { SOCS5, BTG2 } & \text { PTEN/Akt pathway, } \\
\text { PTtch signaling } \\
\text { pathway }\end{array}$ & $\mathrm{Up}$ & Serum & [58] \\
\hline miR-324-3p & $\begin{array}{l}\text { GLI3, WNT2B, } \\
\text { MAPK pathway, } \\
\text { Wnt/ } \beta \text {-catenin } \\
\text { signaling pathway }\end{array}$ & up & Serum & [58] \\
\hline miR-377-3p & $\begin{array}{l}\text { Lysine degradation } \\
\text { and ubiquitin- } \\
\text { mediated } \\
\text { proteolysis } \\
\text { pathways }\end{array}$ & $\mathrm{Up}$ & Serum & [58] \\
\hline miR-378 & & Downregulated & $\begin{array}{l}\text { Plasma- } \\
\text { derived } \\
\text { exosome }\end{array}$ & [73] \\
\hline miR-424-5p & $\mathrm{CDX} 2$ & Downregulated & PBMCs & [72] \\
\hline miR-454-3p & $\begin{array}{l}\text { NFATc2, CALB1, } \\
\text { Wnt/ } \beta- \\
\text { catenin pathway, }\end{array}$ & $\mathrm{Up}$ & Serum & [58] \\
\hline miR-490-5p & $\begin{array}{l}\text { CABP5, PIK3CA, } \\
\text { NFAT5 } \\
\text { TGF-beta- } \\
\text { signaling pathway }\end{array}$ & Downregulated & Serum & [58] \\
\hline miR-574-3p & $\begin{array}{l}\text { SMAD4 } \\
\text { signaling pathway, }\end{array}$ & Downregulated & $\begin{array}{l}\text { Plasma- } \\
\text { derived } \\
\text { exosome }\end{array}$ & [73] \\
\hline
\end{tabular}




\begin{tabular}{|l|l|l|l|l|}
\hline miR-23a-3p & $\begin{array}{l}\text { DP5, PUMA, BAX, } \\
\text { and BIM }\end{array}$ & Downregulated & $\begin{array}{l}\text { Pancreatic } \\
\text { cells }\end{array}$ & {$[60]$} \\
\hline miR-23b-3p & DP5 and PUMA & Downregulated & $\begin{array}{l}\text { Pancreatic } \\
\text { cells }\end{array}$ & {$[60]$} \\
\hline miR-149-5p & $\begin{array}{l}\text { DP5, PUMA, and } \\
\text { BAX }\end{array}$ & Downregulated & $\begin{array}{l}\text { Pancreatic } \\
\text { cells }\end{array}$ & {$[60]$} \\
\hline miR-98 & $\begin{array}{l}\text { TRAIL, TRAIL-R2, } \\
\text { FAS, and FASLG }\end{array}$ & Up & T cells & {$[61]$} \\
\hline miR-146a & $\begin{array}{l}\text { TRAF6, NUMB, } \\
\text { STX3 and BCL11A }\end{array}$ & $\begin{array}{l}\text { Downregulated } \\
\text { FAS, and FASLG }\end{array}$ & PBMCs & {$[72]$} \\
\hline miR-23b & $\begin{array}{l}\text { TRAIL, TRAIL-R2, } \\
\text { FAS, and FASLG }\end{array}$ & Up & T cells & {$[61]$} \\
\hline
\end{tabular}

\section{Targeting the drivers of epigenetic modification in type 1 diabetes}

While it has long been known that environmental factors can increase the risk of type 1 diabetes in susceptible individuals, only recently have insights into cellular metabolism revealed epigenetic modifications as one of the mechanistic links between environmental stimuli and autoimmune disease [74]. This insight has created the field of "metaboloepigenetics"; a transformative framework that is connecting the effects of inflammation and nutritional status to metabolite concentration, which in turn influences the enzymes involved in epigenetic regulation, including DNA methyltransferases and HDACs [74]. Studies have now described several mechanisms that are involved in gene expression regulation and genome stability, which are strongly influenced by dietary nutrients and related metabolites, such as chromatin remodeling, RNA splicing, nuclear RNA export, mRNA stabilization, and non-coding RNA expression [75-78]. Methylation is one of the bestdescribed epigenetic modifications whose effects on genome function and relationship to nutrition and other environmental exposures are beginning to be understood [79]. Such knowledge raises the possibility of "metaboloepigenetic reprogramming": eliminating specific metabolic substrates and managing the accumulation of harmful substances through nutritional and environmental modification is a promising new therapeutic approach to treating complex diseases [80-84]. As such, epigenetic information is a key component of establishing an individual's inflammation-defense blueprint, whereby their autoimmune disease can be treated through a process of individual categorization, biomarker discovery, and personalized therapeutic intervention [77].

\section{Conclusions and future directions}

Diabetes is an expanding global health concern that exerts a considerable economic and quality of life burden on individuals and societies. Refining the biomarkers of early disease or disease risk and identifying those populations that require intensive monitoring or additional treatment is becoming increasingly important. In conjunction with the advent of precision medicine, studies elucidating the relationship between individual genetic variants and specific epigenetic alterations/epigenetic patterns will provide a pathway for developing effective diagnostic approaches and innovative therapeutic strategies for type 1 diabetes.

In the last decade, epigenetics has received great attention as the maestro of the type 1 diabetes orchestra. In this short review, we have described some of the latest discoveries in the field of epigenetics of type 1 diabetes: how interactions between epigenetic modifications and genetic variants can induce type 1 diabetes pathogenesis, how altering metabolite concentrations could erase or generate various epigenetic markers and vice versa, how metabolism and chromatin modifications 
are interconnected, how these processes could significantly influence disease etiology and progression, and how skewing epigenetics can be the key for type 1 diabetes therapy.

Since type 1 diabetes is characterized by altered DNA methylation, histone modifications, and expression of several miRNAs, the treatment of this condition based on the restoration of a physiological epigenetic framework is highly conceivable. However, significant challenges remain. Further future study deigns such as nested case-control studies must be done with people with a known risk factor for type 1 diabetes, as a better understanding of early disease onset will provide insight into the temporal nature of epigenetic alterations and will assess the directionality of their effect - knowledge which is currently lacking. Another important aspect is increasing the sample size to provide adequate power with consideration of ethnicity and population differences. On the other hand, integration of different omics data will give rise to new aspects of the disease, for example: investigating the relationship between DNA methylation, alternative splicing, and metabolism in type 1 diabetes patients. Alongside, some practical barriers need to be overcome, for example how to deliver epigenetic modifiers to the specific cells and tissues of interest, and how to retain them there at therapeutic doses; as well as how to assess the long term risks, such as off-target effects, of these treatments. Overcoming these challenges will require well-designed and ambitious studies that can link in vitro molecular data with in vivo observations in animal models of type 1 diabetes and, finally, with clinical studies in diabetes patients.

\section{References}

1. Patterson, C.C., et al., Worldwide estimates of incidence, prevalence and mortality of type 1 diabetes in children and adolescents: Results from the International Diabetes Federation Diabetes Atlas, 9th edition. Diabetes Research and Clinical Practice, 2019. 157.

2. Pociot, F., Type 1 diabetes genome-wide association studies: not to be lost in translation. Clinical \& Translational Immunology, 2017. 6(12): p. e162.

3. Atkinson, M.A., G.S. Eisenbarth, and A.W. Michels, Type 1 diabetes. Lancet (London, England), 2014. 383(9911): p. 69-82.

4. Cudworth, A.G. and J.C. Woodrow, HL-A ANTIGENS AND DIABETES MELLITUS. The Lancet, 1974. 304(7889): p. 1153.

5. Rojas, J., et al., Pancreatic Beta Cell Death: Novel Potential Mechanisms in Diabetes Therapy. J Diabetes Res, 2018. 2018: p. 9601801.

6. Pociot, F., Type 1 diabetes genome-wide association studies: not to be lost in translation. Clinical \& translational immunology, 2017. 6(12): p. e162-e162.

7. Pociot, F., et al., Genetics of Type 1 Diabetes: WhatE\#039;s Next? Diabetes, 2010. 59(7): p. 1561.

8. Ilonen, J., et al., Rapid HLA-DQB1 genotyping for four alleles in the assessment of risk for IDDM in the Finnish population. The Childhood Diabetes in Finland (DiMe) Study Group. Diabetes Care, 1996. 19(8): p. 795-800.

9. Steck, A.K. and M.J. Rewers, Genetics of type 1 diabetes. Clinical chemistry, 2011. 57(2): p. 176-185.

10. Castillo-Fernandez, J.E., T.D. Spector, and J.T. Bell, Epigenetics of discordant monozygotic twins: implications for disease. Genome medicine, 2014. 6(7): p. 60-60.

11. Egro, F.M., Why is type 1 diabetes increasing? J Mol Endocrinol, 2013. 51(1): p. R1-13.

12. Vehik, K. and D. Dabelea, The changing epidemiology of type 1 diabetes: why is it going through the roof? Diabetes Metab Res Rev, 2011. 27(1): p. 3-13.

13. Gillespie, K.M., et al., Early onset of diabetes in the proband is the major determinant of risk in HLA DR3DQ2/DR4-DQ8 siblings. Diabetes, 2014. 63(3): p. 1041-7.

14. Cerna, M., Epigenetic Regulation in Etiology of Type 1 Diabetes Mellitus. Int J Mol Sci, 2019. 21(1).

15. Jerram, S.T., M.N. Dang, and R.D. Leslie, The Role of Epigenetics in Type 1 Diabetes. Curr Diab Rep, 2017. 17(10): p. 89.

16. Jeltsch, A. and R.Z. Jurkowska, New concepts in DNA methylation. Trends Biochem Sci, 2014. 39(7): p. 310-8.

17. Bansal, A. and S.E. Pinney, DNA methylation and its role in the pathogenesis of diabetes. Pediatr Diabetes, 2017. 18(3): p. 167-177.

18. Rakyan, V.K., et al., Identification of type 1 diabetes-associated DNA methylation variable positions that precede disease diagnosis. PLoS Genet, 2011. 7(9): p. e1002300.

19. Stefan, M., et al., DNA methylation profiles in type 1 diabetes twins point to strong epigenetic effects on etiology. J Autoimmun, 2014. 50: p. 33-7. 
20. Paul, D.S., et al., Increased DNA methylation variability in type 1 diabetes across three immune effector cell types. Nat Commun, 2016. 7: p. 13555.

21. Olsson, A.H., et al., Genome-Wide Associations between Genetic and Epigenetic Variation Influence mRNA Expression and Insulin Secretion in Human Pancreatic Islets. PLOS Genetics, 2014. 10(11): p. e1004735.

22. Ye, J., et al., Identification of loci where DNA methylation potentially mediates genetic risk of type 1 diabetes. bioRxiv, 2018: p. 248260.

23. Johnson, R.K., et al., Longitudinal DNA methylation differences precede type 1 diabetes. Scientific Reports, 2020. 10(1): p. 3721.

24. Lefebvre, B., et al., 5'-AZA induces Ngn3 expression and endocrine differentiation in the PANC-1 human ductal cell line. Biochem Biophys Res Commun, 2010. 391(1): p. 305-9.

25. Chakravarthy, H., et al., Converting Adult Pancreatic Islet alpha Cells into beta Cells by Targeting Both Dnmt1 and Arx. Cell Metab, 2017. 25(3): p. 622-634.

26. Bridgeman, S.C., et al., Epigenetic effects of metformin: From molecular mechanisms to clinical implications. Diabetes Obes Metab, 2018. 20(7): p. 1553-1562.

27. Petrie, J.R., et al., Metformin in adults with type 1 diabetes: Design and methods of REducing with MetfOrmin Vascular Adverse Lesions (REMOVAL): An international multicentre trial. Diabetes Obes Metab, 2017. 19(4): p. 509-516.

28. Luo, F., et al., Metformin in patients with and without diabetes: a paradigm shift in cardiovascular disease management. Cardiovascular Diabetology, 2019. 18(1): p. 54.

29. Livingstone, R., et al., A new perspective on metformin therapy in type 1 diabetes. Diabetologia, 2017. 60(9): p. 1594-1600.

30. García-Calzón, S., et al., Diabetes medication associates with DNA methylation of metformin transporter genes in the human liver. Clinical Epigenetics, 2017. 9(1): p. 102.

31. El-Hadidy, W.F., A.R. Mohamed, and H.F. Mannaa, Possible protective effect of procainamide as an epigenetic modifying agent in experimentally induced type 2 diabetes mellitus in rats. Alexandria Journal of Medicine, 2015. 51(1): p. 65-71.

32. Sato, T., J.-P.J. Issa, and P. Kropf, DNA Hypomethylating Drugs in Cancer Therapy. Cold Spring Harbor perspectives in medicine, 2017. 7(5): p. a026948.

33. Handy, D.E., R. Castro, and J. Loscalzo, Epigenetic modifications: basic mechanisms and role in cardiovascular disease. Circulation, 2011. 123(19): p. 2145-2156.

34. Kouzarides, T., Chromatin modifications and their function. Cell, 2007. 128(4): p. 693-705.

35. Miao, F., et al., Evaluating the role of epigenetic histone modifications in the metabolic memory of type 1 diabetes. Diabetes, 2014. 63(5): p. 1748-1762.

36. Puthanmadhom Narayanan, S., et al., Epigenetic Alterations Are Associated With Gastric Emptying Disturbances in Diabetes Mellitus. Clinical and translational gastroenterology, 2020. 11(3): p. e00136e00136.

37. Martin, C. and Y. Zhang, The diverse functions of histone lysine methylation. Nature reviews Molecular cell biology, 2005. 6(11): p. 838-849.

38. Strahl, B.D. and C.D. Allis, The language of covalent histone modifications. Nature, 2000. 403(6765): p. 41-5.

39. Cao, J. and Q. Yan, Histone ubiquitination and deubiquitination in transcription, DNA damage response, and cancer. Frontiers in oncology, 2012. 2: p. 26-26.

40. Miao, F., et al., Lymphocytes From Patients With Type 1 Diabetes Display a Distinct Profile of Chromatin Histone H3 Lysine 9 Dimethylation. Diabetes, 2008. 57(12): p. 3189.

41. Miao, F., et al., Profiles of epigenetic histone post-translational modifications at type 1 diabetes susceptible genes. Journal of Biological Chemistry, 2012.

42. Wang, Z., et al., Beyond Genetics: What Causes Type 1 Diabetes. Clin Rev Allergy Immunol, 2017. 52(2): p. 273-286.

43. Miao, F., et al., Lymphocytes from patients with type 1 diabetes display a distinct profile of chromatin histone H3 lysine 9 dimethylation: an epigenetic study in diabetes. Diabetes, 2008. 57(12): p. 3189-98.

44. Miao, F., et al., Profiles of epigenetic histone post-translational modifications at type 1 diabetes susceptible genes. J Biol Chem, 2012. 287(20): p. 16335-45.

45. Caramori, M.L., et al., Gene expression differences in skin fibroblasts in identical twins discordant for type 1 diabetes. Diabetes, 2012. 61(3): p. 739-744.

46. Lundh, M., et al., Histone deacetylases 1 and 3 but not 2 mediate cytokine-induced beta cell apoptosis in INS-1 cells and dispersed primary islets from rats and are differentially regulated in the islets of type 1 diabetic children. Diabetologia, 2012. 55(9): p. 2421-31. 
47. Christensen, D.P., et al., Lysine deacetylase inhibition prevents diabetes by chromatin-independent immunoregulation and $\beta$-cell protection. Proceedings of the National Academy of Sciences of the United States of America, 2014. 111(3): p. 1055-1059.

48. Khan, S. and G.B. Jena, Protective role of sodium butyrate, a HDAC inhibitor on beta-cell proliferation, function and glucose homeostasis through modulation of p38/ERK MAPK and apoptotic pathways: study in juvenile diabetic rat. Chem Biol Interact, 2014. 213: p. 1-12.

49. Khan, S. and G. Jena, Valproic Acid Improves Glucose Homeostasis by Increasing Beta-Cell Proliferation, Function, and Reducing its Apoptosis through HDAC Inhibition in Juvenile Diabetic Rat. J Biochem Mol Toxicol, 2016. 30(9): p. 438-46.

50. Grammatiki, M., et al., Vitamin D and diabetes mellitus: Causal or casual association? Reviews in Endocrine and Metabolic Disorders, 2017. 18(2): p. 227-241.

51. Martín-Subero, J.I. and M. Esteller, Profiling Epigenetic Alterations in Disease, in Epigenetic Contributions in Autoimmune Disease, E. Ballestar, Editor. 2011, Springer US: Boston, MA. p. 162-177.

52. $\mathrm{Xu}, \mathrm{C} .-\mathrm{R}$. , et al., Dynamics of genomic H3K27me3 domains and role of EZH2 during pancreatic endocrine specification. The EMBO Journal, 2014. 33(19): p. 2157-2170.

53. Fontcuberta-PiSunyer, M., et al., Modulation of the endocrine transcriptional program by targeting histone modifiers of the H3K27me3 mark. Biochim Biophys Acta Gene Regul Mech, 2018. 1861(5): p. 473-480.

54. Katz, L.S., E. Geras-Raaka, and M.C. Gershengorn, Reprogramming adult human dermal fibroblasts to isletlike cells by epigenetic modification coupled to transcription factor modulation. Stem Cells Dev, 2013. 22(18): p. 2551-60.

55. Marino, E., et al., Gut microbial metabolites limit the frequency of autoimmune $T$ cells and protect against type 1 diabetes. Nat Immunol, 2017. 18(5): p. 552-562.

56. Joglekar, M.V., V.S. Parekh, and A.A. Hardikar, New pancreas from old: microregulators of pancreas regeneration. Trends Endocrinol Metab, 2007. 18(10): p. 393-400.

57. Miao, C., et al., MicroRNAs in type 1 diabetes: new research progress and potential directions. Biochemistry and cell biology = Biochimie et biologie cellulaire, 2018. 96(5): p. 498-506.

58. Erener, S., et al., Profiling of circulating microRNAs in children with recent onset of type 1 diabetes. JCI Insight, 2017. 2(4): p. e89656.

59. Kim, K.W., et al., Coxsackievirus B5 Infection Induces Dysregulation of microRNAs Predicted to Target Known Type 1 Diabetes Risk Genes in Human Pancreatic Islets. Diabetes, 2016. 65(4): p. 996-1003.

60. Grieco, F.A., et al., MicroRNAs miR-23a-3p, miR-23b-3p, and miR-149-5p Regulate the Expression of Proapoptotic BH3-Only Proteins DP5 and PUMA in Human Pancreatic beta-Cells. Diabetes, 2017. 66(1): p. 100-112.

61. de Jong, V.M., et al., Survival of autoreactive T lymphocytes by microRNA-mediated regulation of apoptosis through TRAIL and Fas in type 1 diabetes. Genes Immun, 2016. 17(6): p. 342-8.

62. Krischer, J.P., et al., The influence of type 1 diabetes genetic susceptibility regions, age, sex, and family history on the progression from multiple autoantibodies to type 1 diabetes: a TEDDY study report. Diabetes, 2017. 66(12): p. 3122-3129.

63. Osmai, M., et al., MicroRNAs as regulators of beta-cell function and dysfunction. Diabetes/Metabolism Research and Reviews, 2016. 32(4): p. 334-349.

64. Roggli, E., et al., Involvement of microRNAs in the cytotoxic effects exerted by proinflammatory cytokines on pancreatic beta-cells. Diabetes, 2010. 59(4): p. 978-86.

65. Lovis, P., et al., Alterations in microRNA expression contribute to fatty acid-induced pancreatic beta-cell dysfunction. Diabetes, 2008. 57(10): p. 2728-36.

66. Pileggi, A., et al., MicroRNAs in islet immunobiology and transplantation. Immunol Res, 2013. 57(1-3): p. 185-96.

67. Wang, P., et al., miR-216a-targeting theranostic nanoparticles promote proliferation of insulin-secreting cells in type 1 diabetes animal model. Sci Rep, 2020. 10(1): p. 5302.

68. Chen, H., et al., Application of microRNAs in diabetes mellitus. 2014. 222(1): p. R1.

69. Lin, J., C.-C. Chuang, and L. Zuo, Potential roles of microRNAs and ROS in colorectal cancer: diagnostic biomarkers and therapeutic targets. Oncotarget, 2017. 8(10): p. 17328-17346.

70. Santos, A.S., et al., Increased Expression of Circulating microRNA 101-3p in Type 1 Diabetes Patients: New Insights Into miRNA-Regulated Pathophysiological Pathways for Type 1 Diabetes. Frontiers in Immunology, 2019. 10(1637).

71. Satake, E., et al., Circulating miRNA Profiles Associated With Hyperglycemia in Patients With Type 1 Diabetes. Diabetes, 2018. 67(5): p. 1013-1023.

72. Wang, G., et al., Decreased expression of miR-150, miR146a and miR424 in type 1 diabetic patients: Association with ongoing islet autoimmunity. Biochem Biophys Res Commun, 2018. 498(3): p. 382-387. 
73. Garcia-Contreras, M., et al., Plasma-derived exosome characterization reveals a distinct microRNA signature in long duration Type 1 diabetes. Sci Rep, 2017. 7(1): p. 5998.

74. Wang, Z., et al., Crosstalk between metabolism and epigenetic modifications in autoimmune diseases: a comprehensive overview. Cell Mol Life Sci, 2018. 75(18): p. 3353-3369.

75. Van Rooij, E., et al., The role of microRNA in nutritional control. Journal of Internal Medicine, 2015. 278(2): p. 99-109.

76. Guay, C. and R. Regazzi, Circulating microRNAs as novel biomarkers for diabetes mellitus. Nature Reviews Endocrinology, 2013. 9(9): p. 513.

77. Kilberg, M.S., et al., Nutritional control of gene expression: how mammalian cells respond to amino acid limitation. Annual review of nutrition, 2005. 25: p. 59-85.

78. Mazzone, R., et al., The emerging role of epigenetics in human autoimmune disorders. Clinical Epigenetics, 2019. 11(1): p. 34.

79. Stover, P.J., et al., Emerging concepts on the role of epigenetics in the relationships between nutrition and health. Journal of Internal Medicine, 2018. 284(1): p. 37-49.

80. Guma, M., S. Tiziani, and G.S. Firestein, Metabolomics in rheumatic diseases: desperately seeking biomarkers. Nature Reviews Rheumatology, 2016. 12(5): p. 269-281.

81. Galluzzi, L., et al., Metabolic targets for cancer therapy. Nature Reviews Drug Discovery, 2013. 12(11): p. 829-846.

82. Kinnaird, A., et al., Metabolic control of epigenetics in cancer. Nature Reviews Cancer, 2016. 16(11): p. 694707.

83. Miranda-Gonçalves, V., et al., Metabolism and Epigenetic Interplay in Cancer: Regulation and Putative Therapeutic Targets. Frontiers in genetics, 2018. 9: p. 427-427.

84. Wang, Y.-P. and Q.-Y. Lei, Metabolic recoding of epigenetics in cancer. Cancer Communications, 2018. 38(1): p. 25. 